\title{
Common coding in pigeons: Partial versus total reversals of one-to-many conditional discriminations
}

\author{
THOMAS R. ZENTALL, LOU M. SHERBURNE, JANICE N. STEIRN, \\ CHRISTOPHER K. RANDALL, and KAREN L. ROPER \\ University of Kentucky, Lexington, Kentucky \\ and \\ PETER J. URCUIOLI \\ Purdue University, West Lafayette, Indiana
}

\begin{abstract}
Common coding in pigeons was examined using a delayed conditional discrimination in which each sample stimulus was associated with two different comparison stimuli (one-to-many mapping). In Experiment 1, pigeons matched circle and dot samples to red and green hues and vertical and horizontal line orientations. In Experiment 2, the samples were red and green and the comparisons were vertical and horizontal spatial positions (up vs. down and left vs. right). Following acquisition to high levels of accuracy in each experiment, the associations between the samples and either both sets or only one set of comparisons were reversed. Pigeons learned the total reversals faster than the partial reversals. These results suggest that when different comparisons are associated with a common sample, they may become functionally equivalent.
\end{abstract}

There is growing evidence that when pigeons are trained on discriminations in which more than one stimulus is associated with a common event, those stimuli become functionally equivalent. Not only are the stimuli interchangeable with one another within the stimulus context, but more importantly, they also appear to substitute for one another in other contexts.

An example of this sort of substitutability (also referred to as common coding) was provided by Edwards, Jagielo, Zentall, and Hogan (1982). In this experiment, Edwards et al. trained pigeons on two independent identitymatching tasks, one involving hue matching and the other involving shape matching. Correct matches of one hue and one shape were reinforced with corn, whereas correct matches of the other hue and shape were reinforced with wheat. On transfer trials in which the shapes were substituted as samples in the hue-comparison task, and vice versa, pigeons reliably chose the comparison associated with the same outcome as the sample. Thus, sample stimuli associated with the same outcome had become interchangeable.

Using a different procedure, Vaughan (1988) demonstrated that pigeons could also form stimulus classes based

This research was supported by National Science Foundation Grants BNS-8418275 and BNS-9019080 to T.R.Z. and P.J.U., by National Science Foundation Grant RII 8902792 to J.N.S., and by a grant from the University of Kentucky Research Foundation to T.R.Z. J. Steim is now at the Department of Psychology, Georgia Southern University. Correspondence concerning this article should be sent to T. R. Zentall, Department of Psychology, University of Kentucky, Lexington, KY 40506. on stimuli that, at any given time, were treated in the same way. Vaughan trained pigeons to respond to a set of 20 randomly selected natural stimuli $(S+s)$ and to not respond to a second set of 20 natural stimuli $(S-s)$. Following acquisition, the contingencies associated with both sets were reversed, and then reversed again repeatedly. After a large number of reversals, pigeons responded correctly to stimuli presented later in a session once they had been exposed to a few stimuli from each set early in that session. In other words, their go-versus-no-go response pattern to individual stimuli in each set changed prior to their actually experiencing the new associations with reinforcement and nonreinforcement. Thus, performance with stimuli presented later in the session was controlled by the contingency currently associated with each stimulus set.

A number of procedures have been used to study stimulus class formation in humans. Spradlin, Cotter, and Baxley (1973, Experiment 2), for example, trained subjects that in the presence of either stimulus A or stimulus B, a response to stimulus $C$ would be reinforced. They were then trained that in the presence of stimulus $\mathrm{A}$, a response to stimulus $D$ would be reinforced. Finally, on probe trials with stimulus B, 2 out of 3 subjects responded immediately with a response to stimulus D. Spradlin et al. concluded that "if one member of a stimulus class is conditioned to control a new response, other members of that stimulus class may also control that new response" (p. 564).

This procedure, in which multiple stimuli are associated with a common response (sometimes referred to as manyto-one mapping), has also been shown to produce a simi- 
lar transfer effect in pigeons (Urcuioli, Zentall, JacksonSmith, \& Steirn, 1989, Experiment 2). In this experiment, pigeons were initially trained on a delayed conditional discrimination in which one hue (e.g., red) and one lineorientation sample (e.g., vertical) were associated with one comparison stimulus (e.g., vertical), and the other hue (e.g., green) and line-orientation sample (e.g., horizontal) were associated with the other comparison stimulus (e.g., horizontal). In a second phase of training, the hue samples from this many-to-one task were paired with a new set of comparisons (e.g., red with circle and green with dot). Then, in testing, the remaining line samples were paired for the first time with the new comparisons. When the correct comparison response was consistent with the presumed stimulus sets developed in Phase 1, transfer performance was significantly better than when that response was inconsistent with those stimulus sets (i.e., in the example given, they tended to respond to the circle comparison when the sample was vertical and to the dot comparison when the sample was horizontal).

If sample stimuli that are associated with the same comparison response become commonly coded or are functionally equivalent to each other (i.e., they are interchangeable), then Vaughan's (1988) reversal data suggest that it should be easier to reverse the comparison associations to both samples than to reverse the associations developed to only one of the samples.

In support of this common-coding hypothesis, Zentall, Steirn, Sherburne, and Urcuioli (1991; see also Nakagawa, 1986) showed that following many-to-one training in which red $(R)$ and vertical-line $(V)$ samples were associated with a circle (C) comparison and green (G) and horizontal-line $(\mathrm{H})$ samples were associated with a dot (D) comparison, pigeons acquired the reversal task faster when both sets of associations were reversed (e.g., R and $V$ samples now associated with $D$ comparisons and $G$ and $H$ samples now associated with $C$ comparisons) than when only one set was reversed (e.g., $R$ and $G$ samples now associated with $\mathrm{D}$ and $\mathrm{C}$ comparisons, respectively, but $\mathrm{V}$ and $\mathrm{H}$ samples still associated with $\mathrm{C}$ and $\mathrm{D}$ comparisons, respectively).

The purpose of the experiments presented here was to determine whether evidence for common coding could be found after training in which different comparisons were associated with a common sample. In other words, in a one-to-many conditional discrimination, are the comparisons commonly coded as the samples apparently are in a many-to-one task? If so, then the subsequent reversal of associations between both sets of comparisons and their samples should produce faster relearning than reversing only one set of associations.

Alternatively, if common coding does not occur, and if the rate of reversal learning depends upon the number of associations that must be reversed, then reversal of associations between both sets of comparisons and their samples should produce slower relearning than reversing only one set of associations.

\section{EXPERIMENT 1}

In the first experiment, pigeons were trained to associate two samples (C and D) with two pairs of comparisons ( $R$ and $G ; V$ and $H$ ). These were the sample stimuli used by Zentall et al. (1991), except that the sample and comparison sets were switched. After reaching criterion levels of accuracy on this one-to-many task, the sample associations involving hue comparisons were reversed for Group Hue, whereas the corresponding associations involving the line comparisons were reversed for Group Line. For Group Hue-Line, the sample associations involving both sets of comparisons were reversed. If pigeons learned to commonly code the two comparisons associated with the same sample during initial training, then reversing both associations ought to be easier than reversing only one because the common code could be maintained in the former case, but it would be violated in the latter.

\section{Method}

\section{Subjects}

The subjects were 18 mixed-sex, experimentally naive White Carneau pigeons purchased as retired breeders (over 5 years old) from the Palmetto Pigeon Plant (Sumter, SC). They were housed in individual cages and maintained throughout the experiment at $75 \%-80 \%$ of their free-feeding body weights. They had continuous access to water and grit in their home cages, which were located in a colony room with a 12:12-h light:dark cycle.

\section{Apparatus}

The experiment was conducted in a sound-attenuated test chamber with a three-key intelligence panel. The pigeon compartment in the test chamber measured $37 \mathrm{~cm}$ high $\times 30 \mathrm{~cm}$ wide $\times 34 \mathrm{~cm}$ deep (across the intelligence panel). Three rectangular response keys ( $3.2 \mathrm{~cm}$ wide $\times 2.5 \mathrm{~cm}$ high) were mounted side by side on the panel with their bottom edges $16.0 \mathrm{~cm}$ above the wire-mesh floor of the chamber. Behind each key was a 12-stimulus in-line projector (Industrial Electronic Engineering, Series 10, with G.E. No. 1820 lamps) that projected $R$ or $G$ hues (Kodak Wratten filters Nos. 26 and 60 ) and three white $V$ or $H$ stimuli (each $13 \mathrm{~mm}$ long $x$ $3 \mathrm{~mm}$ wide and separated by $3 \mathrm{~mm}$ ) on the two side keys and a white line-drawn circle $(C ; 16 \mathrm{~mm}$ outside diameter, $13 \mathrm{~mm}$ inside diameter) or a white dot ( $\mathrm{D} ; 5 \mathrm{~mm}$ diameter) on the center key. All lines and shapes were presented on a black background. The opening to the rear-mounted grain feeder was centered on the intelligence panel and was located midway between the bottom of the response keys and the floor. Reinforcement consisted of 2-sec access to Purina Pro Grains. A shielded houselight located $7.6 \mathrm{~cm}$ above the center key provided general chamber illumination. White noise and an exhaust fan provided sound masking. The experiment was controlled by a microcomputer located in an adjacent room.

\section{Procedure}

Pretraining. All pigeons were trained to eat from the grain feeder and were then shaped by the method of successive approximations to peck a white illuminated center key. Once keypecking was established, the stimulus on the center key was changed to C or D (randomly determined) and changed again following each reinforcement. Each stimulus was presented 24 times, with successive stimulus presentations separated by a 10 -sec intertrial interval (ITI). Over the next 2 days, the number of pecks required for reinforcement was increased, first to 5 and then to 10 . On the final two 24- 
Table 1

Design of Experiment 1

\begin{tabular}{cccc}
\hline Phase 1 & \multicolumn{3}{c}{ Phase 2 } \\
\cline { 2 - 4 } All Groups & Group Hue & Group Line & Group Hue-Line \\
\hline C-R & C-G & C-R & C-G \\
D-G & D-R & D-G & D-R \\
C-V & C-V & C-H & C-H \\
D-H & D-H & D-V & D-V \\
\hline
\end{tabular}

Note-The first letter of each pair represents the sample $(\mathrm{C}=$ circle; $\mathrm{D}=\mathrm{dot})$, and the second letter represents the correct comparison $(\mathrm{R}=$ red; $\mathrm{G}=$ green; $\mathrm{V}=$ verical; $\mathrm{H}=$ horizontal).

trial pretraining sessions, the left or right key was lit with $R, G$, $\mathrm{V}$, or $\mathrm{H}$, and a single peck to the lit key resulted in reinforcement.

Phase 1 training. On the day after pretraining, all pigeons began training on a 0 -sec-delay one-to-many, conditional discrimination with randomly alternating $C$ and $D$ samples and with $R$ and $\mathrm{G}$ comparisons on half the trials and $\mathrm{V}$ and $\mathrm{H}$ comparisons on the remaining trials. The hue and line designated as the correct comparison for each sample was counterbalanced across pigeons. Each conditional discrimination trial began with the onset of one of the sample stimuli (C or D) on the center key. Ten pecks to the sample turned it off and immediately produced two comparison stimuli (either $\mathrm{R}$ and $\mathrm{G}$ or $\mathrm{V}$ and $\mathrm{H}$ ) on the adjacent side keys. A single peck to either comparison stimulus turned off both side keys and initiated a 10-sec ITI, during which the houselight was lit. If the comparison-choice response was correct, it was followed by reinforcement during the first $2 \mathrm{sec}$ of the ITI; otherwise, it was followed by the ITI alone.

Training sessions consisted of 96 trials each and were conducted 6 days a week. Each session was counterbalanced for sample stimulus, position of the correct comparison key, and comparison dimension (hues vs. lines). Trial types were presented randomly with the constraint that on no more than 3 consecutive trials could (1) a particular sample stimulus appear, (2) the position of the correct comparison be the same, and (3) the comparison dimension be the same. Each pigeon was trained on the one-to-many task to a criterion of two consecutive sessions at $90 \%$ correct overall and at least $83 \%$ correct on each of the four trial types. Once this criterion was reached, each pigeon was overtrained for an additional 20 sessions. One pigeon was dropped from the study for failure to reach criterion within 60 sessions.

Phase 2 transfer. As the pigeons completed Phase 1, they were assigned to one of three groups: Group Hue, Group Line, or Group Hue-Line. At least 1 pigeon from each of the Phase 1 counterbalancing groups was assigned to each of the Phase 2 groups. For the pigeons in Group Hue, the sample/hue-comparison associations were reversed. For the pigeons in Group Line, the sample/linecomparison associations were reversed. Finally, for the pigeons in Group Hue-Line, all sample/comparison associations were reversed. Reversal contingencies for the three groups, together with a representative training condition, are presented in Table 1 . In all other respects, the procedure used during Phase 2 was the same as that used during Phase 1 . Phase 2 continued for each pigeon until it reached a performance criterion of $80 \%$ correct, or better, on each pair of reversed associations.

\section{Results}

\section{Phase 1}

The pigeons acquired the Phase 1 one-to-many conditional discrimination in an average of 30.8 sessions. Although the three Phase 2 groups did not differ significantly in the rate at which they acquired the Phase 1 task $(F<$ $1)$, the hue-comparison portion of the Phase 1 task was acquired to a criterion of $90 \%$ correct in significantly fewer sessions (16.8) than the line-comparison portion (23.2) $[F(1,16)=8.53]$. The .05 level of significance was used in these and all subsequent analyses.

\section{Phase 2}

One pigeon in Group Hue-Line was dropped from the analysis for failure to reach the Phase 2 criterion (80\% correct) in 70 transfer sessions. This pigeon developed a strong position response that did not change over the 70 transfer sessions. Seventy sessions was more than twice the number required to reach criterion for the next slowest pigeon in that group and more than 1.5 times the number required for the next slowest pigeon in the other two groups.

Because there were differences in the rate of acquisition of the hue-versus line-comparison portions of the Phase 1 task, acquisition of the Phase 2 reversal by each partial reversal group (Groups Hue and Line) was compared with acquisition of the corresponding reversal in Group Hue-Line.

The reversal data, summarized in Figure 1, show that the hue-comparison reversal was accomplished in fewer sessions by pigeons in Group Hue-Line than by pigeons in Group Hue (17.5 vs. 26.2 sessions, respectively) and that the line-comparison reversal was likewise accomplished in fewer sessions by pigeons in Group Hue-Line than by pigeons in Group Line (20.3 vs. 33.7 sessions, respectively).

An analysis of variance (ANOVA) performed on the sessions-to-80\%-correct data for the line-comparison reversal indicated that this reversal was learned significantly faster by Group Hue-Line than by Group Line $[F(1,8)=$ 5.82]. A similar ANOVA performed on the correspond-

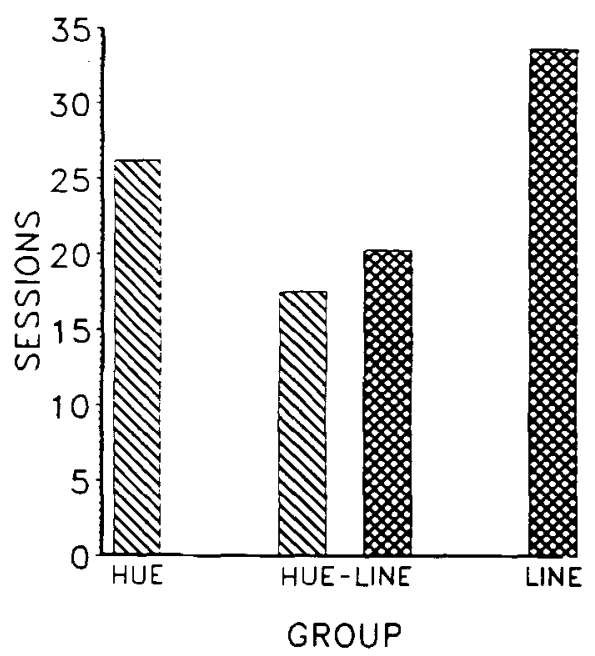

Figure 1. Experiment 1: Mean sessions to $80 \%$ correct on Phase 2 reversals for the hue-comparison conditional discrimination for Group Hue, the line-comparison discrimination for Group Line, and both the hue- and the line-comparison discriminations for Group Hue-Line. 
ing hue-comparison data did not yield a significant effect $[F(1,8)=1.20]$. The reason for this absence of statistical significance appeared to be an unusual amount of within-group variance in Group Hue (range: 7-41 sessions). Note, however, that the percentage increase in sessions to criterion for Group Hue relative to comparable data from Group Hue-Line (50\%) is similar to the percentage increase for Group Line relative to comparable data from Group Hue-Line (66\%). Furthermore, if one reduces the impact of outliers by considering median sessions to criterion, the difference in rate of acquisition between Group Hue (median $=27.5$ sessions) and the hue portion of the task for Group Hue-Line (median $=15.0$ sessions) is even larger (an increase of $83 \%$ ). This contrast is quite comparable to the difference in median rate of acquisition between Group Line (median $=34.0$ sessions) and the line portion of the task for Group HueLine (median $=18.0$ sessions), an increase of $89 \%$. Thus, in spite of the difference in statistical significance between the partial versus total reversal involving lines and analogous comparison involving hues, we believe that it may be more appropriate to conclude that similar retarded acquisition effects occurred in both partial-reversal groups.

As in Phase 1, the hue-comparison associations were learned faster than the line-comparison associations in Phase 2. This difference was evident in the significant within-group comparison-dimension effect found in Group Hue-Line: sessions to criterion for the hue-comparison portion averaged 17.5 as compared with 20.3 sessions for the line-comparison portion $[F(1,3)=10.37]$. An even larger difference was found when Phase 2 acquisition by pigeons in Group Hue (26.2) was compared with acquisition by pigeons in Group Line (33.7), although this difference did not reach statistical significance $[F(1,10)=$ 1.23]. Again, the large within-group variability in Group Hue was probably responsible for this lack of statistical significance.

\section{Discussion}

The results of this experiment showed that after pigeons are trained on a one-to-many conditional discrimination with shapes as samples and hues and line orientations as comparisons, relearning the sample-comparison associations tends to be slower in the context of a partial reversal than in the context of a total reversal. This finding suggests that one-to-many training results in the common coding of comparisons that are associated with the same sample. Consequently, it was easier for the pigeons to learn that responding to both members of a comparison "set" was reinforced following the alternative sample than it was for them to learn that the membership of the sets themselves had changed.

The present findings were obtained with procedures and stimuli that were the same as those used by Zentall et al. (1991, Experiment 1) to examine common coding in a many-to-one conditional discrimination. The results of the present study confirm and extend the Zentall et al. find- ings, with the exception that Zentall et al. reported no difference in sessions to reverse between their Group Hue partial-reversal group (involving hue-sample/shapecomparison association reversals) and the comparable association reversals for their Group Hue-Line total-reversal group. In the present experiment, no asymmetry was found between line and hue reversals in the magnitude of the partial versus total reversal effect.

\section{EXPERIMENT 2}

The primary purpose of Experiment 2 was to assess the generality of the total-versus partial-reversal effect by training pigeons on a one-to-many task involving hue samples and two spatial dimensions as comparisons. On half of the trials, left and right comparison keys were illuminated by white light, and a response to one or the other was reinforced depending on the identity of the prior sample. On the other half of the trials, keys directly above and below the sample were lit, and a response to either the top or the bottom key was reinforced. Following acquisition to criterion, the pigeons were again tested, this time with either one or both spatial dimensions reversed.

A second purpose of Experiment 2 was to examine differences in the acquisition and reversal of a conditional spatial discrimination involving the horizontal versus the vertical dimension. It has been suggested that for lower animals, as well as humans, discriminations along the horizontal dimension are more difficult than those along the vertical dimension because there is a virtual left-right (but not up-down) symmetry in the natural world (see, e.g., Corballis \& Beale, 1976). For example, a left-right reversal (i.e., rotation around the vertical axis) of a natural scene would be much more difficult to detect than a top-bottom reversal (i.e., rotation around the horizontal axis).

\section{Method}

\section{Subjects}

The subjects were 18 mixed-sex, experimentally naive White Carneau pigeons purchased as retired breeders (over 5 years old) from the Palmetto Pigeon Plant (Sumter, SC). They were housed and maintained as described in Experiment 1.

\section{Apparatus}

The experiment was conducted in a sound-attenuated chamber similar to that used in Experiment 1. The intelligence panel used in Experiment 2 contained a $5 \times 5$ matrix of $1.6-\mathrm{cm}$ round pecking keys, spaced $3.1 \mathrm{~cm}$ apart center to center. The only keys used in this experiment were the middle key in the second and fourth rows (referred to as the top and bottom keys) and the three middle keys in the third row (referred to as the left, center, and right keys). Each key could be back-illuminated by one of two 24-V lamps (Sylvania $28 \mathrm{ESB}$ ). The center-key lamps were fitted with Profax caps to produce $\mathbf{R}$ or $\mathrm{G}$ hues. The remaining lamps (top, bottom, left, and right) were uncovered and produced white light $(W)$. The pigeons had access to a rear-mounted grain feeder through an aperture located in the center of the intelligence panel. The bottom edge of the aperture was $3 \mathrm{~cm}$ from the chamber floor. Reinforcement consisted of $2-\mathrm{sec}$ access to pigeon grain. A shielded houselight 
Table 2

Design of Experiment 2

\begin{tabular}{cccc} 
Phase 1 & \multicolumn{3}{c}{ Phase 2 } \\
\cline { 4 - 4 } All Groups & Group V & Group H & Group V-H \\
\hline R-T & R-B & R-T & R-B \\
G-B & G-T & G-B & G-T \\
R-L & R-L & R-R & R-R \\
G-R & G-R & G-L & G-L \\
\hline
\end{tabular}

Note-The first letter of each pair represents the sample $(R=$ red; $G=$ green), and the second letter represents the correct comparison ( $\mathrm{T}=$ top; $\mathrm{B}=$ bottom; $\mathrm{L}=$ left $\mathrm{R}=$ right). $\mathrm{V}=$ vertical; $\mathbf{H}=$ horizontal

was located in the ceiling of the chamber. White noise and an exhaust fan provided sound masking. The experiment was controlled by a microcomputer located in an adjacent room.

\section{Procedure}

Pretraining. The pigeons were pretrained (according to the procedure described in Experiment 1) to eat from the grain feeder and then to peck $R$ and $G$ stimuli on the center key and the $W$ stimulus on the left, right, top, and bottom keys.

Phase 1 training. On the day following the end of pretraining, all pigeons began training on a 0 -sec-delay one-to-many conditional discrimination with randomly alternating $R$ and $G$ samples presented on the center key and $\mathrm{W}$ comparisons presented either on the left and right keys or on the top and bottom keys. The two positions designated as correct for each of the sample hues (left vs. right and top vs. bottom) were counterbalanced over pigeons. Each trial began with the onset of one of the two hues on the center key. Ten pecks to the sample turned it off and immediately produced two comparison stimuli (the left and right keys or the top and bottom keys). A single peck to either comparison turned both off and initiated a 10-sec ITI, during which the houselight was lit. If the comparison-choice response was correct, it was followed by reinforcement during the first $2 \mathrm{sec}$ of the ITI; otherwise, it was followed by the ITI alone.

As in Experiment 1, training sessions consisted of 96 trials each. Trials within a session were counterbalanced for sample hue, position of the correct comparison key, and comparison dimension. Trials were presented randomly, with the same constraints as in Experiment 1. Again, each pigeon was trained to a performance criterion of two consecutive sessions at $90 \%$ correct overall and $83 \%$ correct, or better, on each of the four trial types. Once this criterion was reached for trials involving each of the spatial dimensions, each pigeon was overtrained for an additional 20 sessions.

Phase 2 transfer. As the pigeons completed Phase 1, they were randomly assigned to one of three groups $(n=6)$ : Group V, for which the sample/comparison-position associations in the vertical dimension were reversed; Group $\mathrm{H}$, for which the comparison associations involving the horizontal dimension were reversed; and Group V-H, for which both were reversed. Reversal contingencies for the three groups, together with representative training conditions, are presented in Table 2 . In all other respects, the procedure used during Phase 2 was the same as that used during Phase 1. Phase 2 continued for each pigeon until it performed at a level of $80 \%$ correct or better on each pair of reversed associations.

\section{Results}

\section{Phase 1}

The pigeons acquired the Phase 1 one-to-many conditional discrimination in an average of 14.9 sessions. The pigeons did not differ significantly in number of sessions to acquire the Phase 1 task, as a function of the Phase 2 group to which they were assigned $(F<1)$. A within- subject analysis indicated that the sample-comparison associations involving the vertical dimension were not learned significantly faster than comparable associations involving the horizontal dimension: 14.2 versus 15.4 sessions, respectively $[F(1,17)=2.35]$.

\section{Phase 2}

The pigeons in Group $V$ acquired the Phase 2 reversal at about the same rate (15.0 sessions) as did the pigeons in Group $\mathrm{H}$ ( 15.5 sessions) $(F<1)$. Furthermore, in Group V-H, reversal of the vertical associations in Phase 2 was not learned significantly faster $(M=11.8$ sessions) than reversal of the horizontal associations ( $M=$ 10.8 sessions) $(F<1)$.

Because the spatial dimension of the two comparison sets did not affect the rate of Phase 2 reversal, the reversal data from the two partial-reversal groups (i.e., Groups $\mathrm{V}$ and $\mathrm{H}$ ) were combined for the purpose of anal$y$ sis and were compared to the average sessions to reverse the vertical-and horizontal-comparison associations in the total-reversal group (i.e., Group V-H). An ANOVA performed on these data indicated that the total-reversal group learned their reversals significantly faster $(M=11.3$ sessions) than did the partial-reversal groups ( $M=15.3$ sessions) $[F(1,16)=5.06]$. Mean Phase 2 reversal performances for the three groups are presented in Figure 2.

\section{Discussion}

The results of Experiment 2 confirm and extend the results of Experiment 1. Following acquisition of a one-tomany conditional discrimination involving hues as samples and vertically versus horizontally displayed spatial

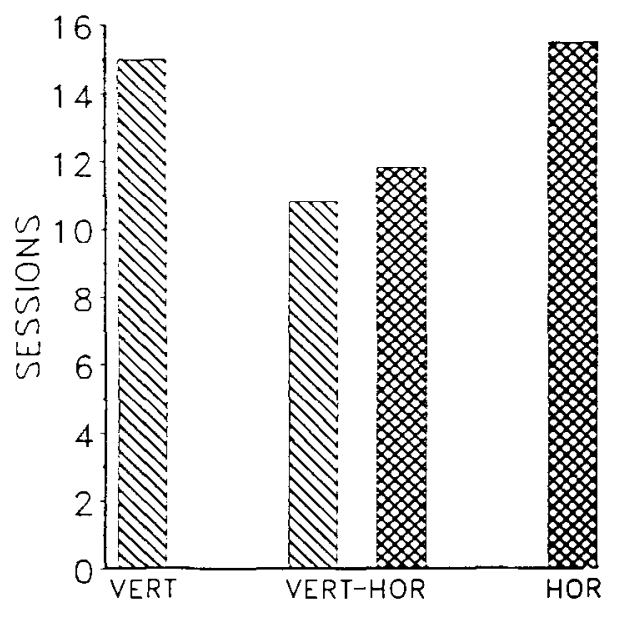

GROUP

Figure 2. Experiment 2. Mean sessions to $80 \%$ correct on Phase 2 reversals for the vertical-comparison conditional discrimination for Group V, the horizontal-comparison discrimination for Group $\mathbf{H}$, and both the vertical- and the horizontal-comparison discriminations for Group V-H. 
comparisons, a total reversal involving both comparison dimensions was learned significantly faster than a partial reversal involving either one or the other dimension alone. These results support the hypothesis that the one-to-many mapping of samples onto comparisons encourages the development of common comparison codes, just as acquisition of a many-to-one conditional discrimination encourages the development of common sample codes.

The results of Experiment 2 also indicate that spatial discriminations involving locations in the vertical dimension were not learned significantly faster than discriminations in the horizontal dimension. Similarly, there were no significant differences in sessions to reverse between discriminations in the vertical versus horizontal dimensions. The absence of an effect of reversal dimension was found both in the between-group comparison involving Group V versus Group $\mathrm{H}$ and in the within-subject comparison involving Group V-H. Apparently, pigeons do not show an asymmetry in either the acquisition or the reversal of spatial-discrimination learning between stimuli presented in the vertical versus the horizontal dimension, as Corballis and Beale (1976) predicted.

The absence of an effect of spatial dimension in the present experiment is consistent with recent findings from a study involving a radial-arm-maze-analogue task with pigeons (Steirn, Zentall, \& Sherburne, 1992). In this task, pigeons were rewarded on each trial for responding to each of five keys, once and only once. For two of the groups in this study, the keys were arranged in a linear array (either vertical or horizontal). The results indicated that the two groups did not differ either in rate of task acquisition or in level of performance when a delay was interpolated at various points in the trial.

Although vertical versus horizontal asymmetries have been found in a number of studies with human subjects, Maki, Grandy, and Hauge (1979) have noted that such asymmetries only occur when verbal responses (e.g., "up," "down," "right," "left") are required. If the difficulty that humans have with the left-right discrimination is related to the ambiguity that comes from the verbal labels "left" and "right," then one would not expect to find such an asymmetry in pigeons.

\section{GENERAL DISCUSSION}

Results of the two experiments presented here suggest that common coding occurs not only when two or more samples are mapped onto a common comparison (as has been reported earlier), but also when two or more comparisons are mapped onto a common sample.

\section{Common Coding Versus Equivalence}

In the present paper, the term common coding has been used to describe results similar to those from stimulusequivalence experiments. Although we believe that similar processes underlie both phenomena, we have chosen to use the term common coding because stimulus equiva- lence often implies findings beyond those that we have observed.

For example, Sidman and Tailby (1982) indicated that the term stimulus equivalence should be reserved for experiments in which one can demonstrate the three defining properties of an equivalence relation: reflexivity (i.e., performance of generalized identity), symmetry (i.e., the demonstration of bidirectional learning or the use of backward associations following training with forward associations), and transitivity (i.e., the ability of one stimulus to mediate the relation between two other stimuli).

Although these three properties of stimulus equivalence have not yet been demonstrated by pigeons in a single experiment, indirect evidence for at least two of these properties, symmetry and transitivity, have been demonstrated by Urcuioli et al. (1989). In that experiment, pigeons were first trained to respond to a particular comparison stimulus (C) when presented with either of two sample stimuli (A or B). Then they were trained to respond to a new comparison (D) when presented with one of those samples (A). Finally, they showed a reliable tendency to respond to comparison D when presented with sample B. Because the only trained relation involving comparison $D$ involved sample $A$, there must have been some relation established between samples $B$ and $A$. But the only relation that could have been established between samples $B$ and $A$ would have to be mediated through comparison $\mathrm{C}$. According to Sidman (1986), the demonstration of this relation between samples B and A, which is mediated through comparison $\mathrm{C}$, provides suggestive evidence for the use of transitivity by pigeons. Furthermore, Sidman also indicted that for comparison $C$ to have mediated the relation between samples B and A, it must have involved a symmetry relation (or a backward association) between sample A and comparison $\mathrm{C}$.

The same reasoning can be applied to the partial-versus total-reversal procedure. In this case, pigeons first learn that, when presented with either sample A or sample B, to respond to comparison $C$ but not to comparison $D$. Then, in the case of the partial reversal, although sample A still indicates that a response to comparison $C$ is correct, sample B now indicates that an incompatible response (i.e., a response to comparison $\mathrm{D}$ ) is correct. Thus, any tendency to maintain the relation between samples $A$ and $B$ would result in retardation of the partial reversal. In the case of the total reversal, however, any tendency to maintain the relation between samples $B$ and $A$ should facilitate acquisition of the reversal task because the reversal tasks for the samples are compatible.

A similar argument can be made for the one-to-many conditional-discrimination task (see, e.g., Sidman, 1986; Sidman \& Tailby, 1982). In this case, a relation is trained between sample A and comparisons B and C. According to Sidman, demonstration of a resulting relation between comparisons $B$ and $C$ suggests transitivity with sample $A$ as a mediator. Sidman would maintain that such a demonstration also suggests symmetry because, in this case, 
transitivity requires that the trained sample $\mathrm{A} /$ comparison $\mathrm{B}$ relation result in a symmetrical comparison $B /$ sample $A$ relation.

Demonstration of the third component of stimulus equivalence, reflexivity (or identity), is a bit more problematic. Although there is growing evidence that pigeons are capable of acquiring a generalized identity rule (Zentall, Edwards, Moore, \& Hogan, 1981; Zentall \& Hogan, 1974, 1975, 1976, 1978), these experiments have not been done in the context of tests for symmetry and transitivity.

According to Sidman (1986), the way to test for reflexivity is to train with a conditional discrimination involving, for example, reinforcement for responding to comparison B when the sample is A and then asking if a response will be made to comparison $B$ when the sample is B. But even if an animal "recognizes" the identity relation between sample $B$ and comparison $B$, is there any reason to expect that a response will be made to comparison $B$ rather than to the other comparison (i.e., in the context of comparison $\mathbf{B}$, the animal may also recognize the oddity relation between the other comparison and sample B)?

In fact, there is some evidence that when pigeons perform either a matching or an oddity task they base their responses not simply on the contingency associated with responding to the correct comparison stimulus (as predicted by Skinner, 1950), but rather on the contingency associated with the comparison stimulus that matches the sample (see Zentall et al., 1981). When, following matching or oddity training, the correct or the incorrect comparison stimulus was replaced by a familiar stimulus, but one that was never experienced in the context of that particular sample, a high level of performance on either task depended only on the presence of a matching comparison stimulus. In the case of the oddity task, this meant that replacing the correct comparison resulted in little effect on the task performance, whereas replacing the incorrect comparison resulted in a substantial decrement in task performance. Thus, it is clear that pigeons use the matching relation between sample and comparison as the basis for both matching- and oddity-task performance and that they pass the reflexivity test.

\section{Failures of Equivalence Relations in Animals}

The successful demonstration of reflexivity (e.g., Zentall et al., 1981), as well as indirect evidence for symmetry (e.g., Urcuioli et al., 1989; Zentall, Sherburne, \& Steirn, 1992) and transitivity (e.g., Steirn, Jackson-Smith, \& Zentall, 1991; Urcuioli et al., 1989) in pigeons (i.e., the defining relations involved in stimulus equivalence), suggest that pigeons may be able to satisfy criteria for the demonstration of equivalence relations. However, results from other animal research suggest that pigeons are unable to demonstrate the transitivity relation (D'Amato, Salmon, Loukas, \& Tomie, 1985; Lipkens, Kop, \& Matthijs, 1988; Richards, 1988) and that monkeys and baboons (D'Amato et al., 1985; Sidman et al., 1982) and pigeons (D’Amato et al., 1985; Hogan \& Zentall, 1977;
Lipkens et al., 1988) are unable to demonstrate the symmetry relation. If the reasons for these failures with animals could be identified, we might be in a better position to determine the boundary conditions under which stimulus equivalence occurs.

One problem with some of the designs that have tested for these emergent relations is that they do not allow the animal experience with the test stimuli in locations or under conditions that are similar to the test locations or conditions. For example, in Hogan and Zentall's (1977) test for symmetry, in which pigeons learned to respond to comparison $B$ when the sample was $A$ and were then tested for their response to comparison $A$ when the sample was B, the A samples had never before appeared as comparisons (see also Richards, 1988). Even when animals have experienced the test stimuli in their test locations, symmetry effects may not be found (Lipkens et al., 1988), but as Lipkens et al. noted, “equivalence relations can fail to emerge because a stimulus presented separately as a sample can be a different stimulus when presented as a comparison in combination with another comparison even though its spatial location remains the same" (p. 406).

Sidman et al. (1982) avoided this problem in experiments with monkeys by training not only with the sample-A/comparison-B relation, but also with identity relations involving both $A$ and $B$ stimuli (i.e., training to respond to comparison $A$ when the sample was $A$ and to respond to comparison $B$ when the sample was $B$ ), prior to the critical sample-B/comparison-A test. Such training should provide the animals with experience with all stimuli in all locations. Nevertheless, Sidman et al. failed to find evidence for symmetry in monkeys. According to Sidman et al., one possibility for this failure may be that samples were always presented on the center key, whereas comparisons were always presented elsewhere. Evidence for the development of functional classes may have been obscured "by confining the potential members of each class to particular locations on the key matrix"' (p. 43).

Alternatively, some aspect of the stimuli or procedure may have prevented the development of equivalence relations. According to Sidman et al. (1982), the development of certain functional classes "may depend on the ethological validity of the stimuli and functions being examined"' (p. 43).

One approach that appears to be successful in developing symmetry relations in pigeons is to use a biologically meaningful event as one of the training stimuli. For example, Zentall et al. (1992) trained pigeons in a conditional-discrimination with differential outcomes (see, e.g., Peterson, 1984): when the sample was A1, a response to comparison B1 was followed by food, but when the sample was A2, a response to comparison B2 was followed by no food. When the pigeons were then presented with food and no-food samples, they showed a significant tendency to respond to comparisons $B 1$ and $B 2$, respectively.

In the case of transitivity tests as well, there is a problem with location of the training stimuli. For example, 
D'Amato et al. (1985) trained pigeons to respond to comparison B when the sample was A and to respond to comparison $C$ when the sample was $B$. They then tested the pigeons for a response to comparison $C$ when the sample was $\mathrm{A}$. The reasoning behind this test is that the B stimulus can act as a mediator between sample $A$ and comparison $\mathrm{C}$. However, this conclusion requires the assumption that without specific training pigeons will treat sample $B$ as equivalent to comparison $B$. But there is reason to believe that, in the case of pigeons, the location of a stimulus forms an integral part of the total stimulus and, thus, B comparisons are not automatically interchangeable with B samples.

One way to deal with this problem is to use food and no-food events as the B stimuli in a transitivity test (see Steirn et al., 1991). If stimulus $A$ is associated with a food outcome and then a food sample is associated with comparison $\mathrm{C}$, the problem with stimulus location is avoided (food always appears at the same location). Furthermore, the use of food and no food as the mediating events may maximize the likelihood of observing transitivity because those events are biologically meaningful to the pigeon.

Another problem in interpreting the results of experiments that have attempted to demonstrate stimulus equivalence in animals arises when the purported stimulus set is produced by requiring animals to respond in a particular way to one set of stimuli and to respond in a different way to another set of stimuli. In other words, if the trained response serves as the common link among the stimuli in a set, there is no need to posit emergent relations to account for test results. Research reported by McIntire, Cleary, and Thompson (1987) demonstrates this problem. McIntire et al. trained monkeys, for example, to respond to sample Al in one way (Type 1) and to respond to sample $A 2$ in another way (Type 2) to produce comparisons $\mathrm{B} 1$ and $\mathrm{B} 2$. In the presence of sample A1, a Type 1 response to comparison $B 1$ was reinforced, whereas in the presence of sample $A 2$, a Type 2 response to comparison B2 was reinforced. Similarly, comparisons B1 and B2 were presented as samples, and Type 1 and 2 responses, respectively, produced comparisons $\mathrm{Cl}$ and $\mathrm{C} 2$. In the presence of sample B1, a Type 1 response to comparison $\mathrm{Cl}$ was reinforced, whereas in the presence of sample B2, a Type 2 response to comparison $\mathrm{C} 2$ was reinforced. On test trials involving $A$ samples and $C$ comparisons, appropriate $\mathrm{C} 1$ and $\mathrm{C} 2$ responding was found when the samples were $\mathrm{A} 1$ and $\mathrm{A} 2$, respectively. The apparent emergence of a transitive relation may reflect nothing more than the trained association between Type 1 and Type 2 responding and choice of comparisons $\mathrm{C} 1$ and $\mathrm{C} 2$, respectively (see also Hayes, 1989).

\section{The Nature of the Common Code}

Comparison of the results of Experiment 1 with those of Zentall et al. (1991, Experiment 1; the same stimuli were used in both experiments) may suggest something about the nature of the common codes used in both procedures. As mentioned earlier, although statistically different results were obtained with the one-to-many proce- dure when performance by the two partial-reversal groups was compared with the appropriate total-reversal control conditions, comparison of the means (see Figure 1) and medians suggests that the two effects were actually quite comparable. On the other hand, examination of the results of Zentall et al. (1991, Experiment 1) suggests that although Group Line took significantly longer to acquire the line-reversal task than did Group Hue-Line, the hue reversals for Group Hue and Group Hue-Line were acquired at virtually the same rate.

Furthermore, the facts that the pattern of results found with the many-to-one task held up when a simple successive discrimination was substituted for the conditional discrimination (Zentall et al., 1991, Experiment 2) and that the pattern of results found in Experiment 1 in the present research with the one-to-many task held up when a conditional spatial discrimination was substituted for the conditional visual discrimination (Experiment 2 ) suggest that the differences between findings obtained with the many-to-one procedure and those obtained with the one-to-many procedure are both reliable and general. Based on the results from both sets of experiments, the most reasonable conclusion is that the nature of the common coding that occurs with the two mapping procedures is somewhat different.

Zentall et al. (1991) argued that the asymmetry found with the many-to-one procedure provides an indication of the nature of the common code. Specifically, given that hue samples are more easily discriminated and remembered than line samples (see Urcuioli \& Zentall, 1986; Zentall, Urcuioli, Jagielo, \& Jackson-Smith, 1989), perhaps pigeons learn to code as hues lines that are associated with the same comparison as those hues. If so, then a reversal involving hue samples would require learning new associations between common codes and comparisons in both partial- and total-reversal groups. By contrast, reversing only the line-sample associations would require a regrouping of the stimuli that are commonly coded. Such regrouping presumably would be more difficult and, thus, should slow the rate of reversal.

In the one-to-many tasks of both experiments reported here, however, we would argue that no corresponding asymmetry was found (i.e., the relative retardation of reversal learning was of comparable magnitude for the two partial-reversal groups). What does this tell us about the nature of the underlying common code with the one-tomany procedure? We propose that it indicates that the common code probably does not consist of an anticipatory code involving the hue comparison. That is, if a circle sample is associated with red and vertical-line comparisons, pigeons probably do not code the circle in terms of a "response instruction" to peck the red comparison. If the pigeons had used such anticipatory hue codes, one would have expected reversal of those codes by Group Hue to have been comparable to that by Group Hue-Line.

On the other hand, it is also clear that pigeons do not simply use memory of the sample code as the basis for learning four separate sample-comparison associations. If they did, there would be no reason to expect that the partial-reversal groups, for which only two of the Phase 1 
associations had to be reversed, would take longer to reverse than the total-reversal group, for which all four of the Phase 1 associations had to be reversed.

Although it may not be possible at this time to identify the nature of the common code used by pigeons in acquisition of the one-to-many conditional discrimination, evidence is accumulating that pigeons do use common codes under a variety of conditions in which the demands of the task encourage such processes. Furthermore, these data add to the growing literature that indicate the pigeon's ability to form stimulus classes consisting of previously unrelated stimuli, based solely on their common association with another arbitrary stimulus, either in the forward direction (in the many-to-one design) or in the backward direction (in the one-to-many design).

\section{REFERENCES}

Corballis, M. C., \& Beale, I. L. (1976). The psychology of lefi and right. Hillsdale, NJ: Erlbaum.

D'amato, M. R., Salmon, D. P., Loukas, E., \& Tomie, A. (1985). Symmetry and transitivity of conditional relations in monkeys ( $\mathrm{Ce}$ bus apella) and pigeons (Columba livia). Journal of the Experimental Analysis of Behavior, 44, 35-48.

Edwards, C. A., Jagielo, J. A., Zentall, T. R., \& Hogan, D. E. (1982). Acquired equivalence and distinctiveness in matching to sample by pigeons: Mediation by reinforcer-specific expectancies. Journal of Experimental Psychology: Animal Behavior Processes, 8, 244-259.

HAYES, S. C. (1989). Nonhumans have not yet shown stimulus equivalence. Journal of the Experimental Analysis of Behavior, 51, 385-392.

Hogan, D. E., \& ZENTALL, T. R. (1977). Backward associations in the pigeon. American Journal of Psychology, 90, 3-15.

LiPKenS, R., KoP, P. F. M., \& MatThiss, W. (1988). A test of symmetry and transitivity in the conditional discrimination performances of pigeons. Journal of the Experimental Analysis of Behavior, 49, 395-410.

Maki, R. H., Grandy, C. A., \& Hauge, G. (1979). Why is telling right from left more difficult than telling above from below? Joumal of Experimental Psychology: Human Perception \& Performance, 5, 52-67.

McIntiRe, K. D., Cleary, J., \& Thompson, T. (1987). Conditional relations by monkeys: Reflexivity, symmetry, and transitivity. Journal of the Experimental Analysis of Behaivor, 47, 279-285.

Nakagawa, E. (1986). Overtraining, extinction, and shift learning in a concurrent discrimination in rats. Quarterly Journal of Experimental Psychology, 38, 313-326.

Peterson, G. B. (1984). How expectancies guide behavior. In H. L. Roitblat, T. G. Bever, \& H. S. Terrace (Eds.), Animal cognition (pp. 135-148). Hillsdale, NJ: Erlbaum.

RICHARDS, R. W. (1988). The question of bidirectional associations in pigeons' learning of conditional discrimination tasks. Bulletin of the Psychonomic Society, 26, 577-579.

SIDMAN, M. (1986). Functional analysis of emergent verbal classes. In T. Thompson \& M. D. Zeiler (Eds.), Analysis and integration of behavioral units (pp. 213-245). Hillsdale, NJ: Erlbaum.

Sidman, M., Rauzin, R., Lazar, R., Cunningham, S., Tailby, W.,
\& Carrigan, P. (1982). A search for symmetry in the conditional discriminations of rhesus monkeys, baboons, and children. Joumal of the Experimental Analysis of Behavior, 37, 23-44.

Sidman, M., \& TaILBY, W. (1982). Conditional discrimination vs matching to sample: An expansion of the testing paradigm. Journal of the Experimental Analysis of Behavior, 37, 5-22.

Skinner, B. F. (1950). Are theories of leaming necessary? Psychological Review, 57, 193-216.

Spradlin, J. E., Cotter, V. W., \& Baxley, N. (1973). Establishing a conditional discrimination without direct training: A study of transfer with retarded adolescents. American Journal of Mental Deficiency, $77,556-566$

Steirn, J. N., Jackson-Smith, P., \& Zentall, T. R. (1991). Mediational use of internal representations of food and no-food events by pigeons. Learning \& Motivation, 22, 353-365.

Steirn, J. N., Zentall, T. R., Sherburne, L. M. (1992). Pigeons performances of a radial-arm-maze analog task: Effect of spatial distinctiveness. Psychological Record, 42, 255-272.

UrCuiol, P. J., \& Zentall, T. R. (1986). Retrospective memory in pigeons' delayed matching-to-sample. Journal of Experimental Psychology: Animal Behavior Processes, 12, 69-77.

Urcuioli, P. J., Zentall, T. R., Jackson-Smith, P., \& Steirn, J. N. (1989). Evidence for common coding in many-to-one matching: Retention, intertrial interference, and transfer. Joumal of Experimental Psychology: Animal Behavior Processes, 15, 264-273.

VAUGHaN, W., JR. (1988). Formation of equivalence sets in pigeons Joumal of Experimental Psychology: Animal Behavior Processes, 14. $36-42$.

Zentall, T. R., Edwards, C. A., Moore, B. S., Hogan, D. E. (1981). Identity: The basis for both matching and oddity learning in pigeons. Journal of Experimental Psychology: Animal Behavior Processes, 7, 70-86.

Zentall, T. R., Hogan, D. E. (1974). Abstract concept learning in the pigeon. Journal of Experimental Psychology, 102, 393-398.

Zentall, T. R., Hogan, D. E. (1975). Concept learning in the pigeon: Transfer of matching and nonmatching to new stimuli. American Journal of Psychology, 88, 233-244.

Zentall, T. R., \& Hogan, D. E. (1976). Pigeons can learn identity, difference, or both. Science, 191, 408-409.

Zentall, T. R., \& Hogan, D. E. (1978). Same/different concept leaming in the pigeon: The effect of negative instances and prior adaptation to the transfer stimuli. Journal of the Experimental Analysis of Behavior, 30, 177-186.

Zentall, T. R., Sherburne, L. M., Steirn, J. N. (1992). Development of excitatory backward associations during the establishment of forward associations in a delayed conditional discrimination by pigeons. Animal Learning \& Behavior, 20, 199-206.

Zentall, T. R., Steirn, J. N., Sherburne, L. M., Urcuiol, P. J. (1991). Common coding in pigeons assessed through partial versus total reversals of many-to-one conditional and simple discriminations. Journal of Experimental Psychology: Animal Behavior Processes, 17, 194-201.

Zentall, T. R., Urcuiol, P. J., Jagielo, J. A., \& Jackson-Smtth, P. (1989). Interaction of sample dimension and sample-comparison mapping on pigeons' performance of delayed conditional discriminations. Animal Learning \& Behavior, 17, 172-178.

(Manuscript received July 12, 1991; revision accepted for publication April 6, 1992.) 